\title{
Aphyllophoroid fungi (Basidiomycota) of the middle part of Yenisei River basin, East Siberia, Russia
}

\author{
ANTON G. SHIRYAEV and HEIKKI KOTIRANTA
}

\begin{abstract}
SHIRYAEV, A.G. and KOTIRANTA, H. 2015 (2016): Aphyllophoroid fungi (Basidiomycota) of the middle part of Yenisei River basin, East Siberia, Russia - Karstenia 55: 43-60. HELSINKI. ISSN 0453-3402.

Species assemblage and taxonomical notes of some aphyllophoroid fungi of the middle part of Yenisei river basin are provided. The material was collected in August 2013. Altogether 211 species belonging to 94 genera are reported. The three study localities are situated in the middle boreal subzone in East Siberia, and comparisons with some other Siberian Nature Reserves in the middle boreal subzone are made. Some threatened species are briefly discussed and a few notes on interesting specimens are given.
\end{abstract}

Key words: aphyllophoroid fungi, East Siberia, threatened species

Anton G. Shiryaev, Institute of Plant and Animal Ecology, Vegetation and Mycobiota Biodiversity Department, Ural Division of the Russian Academy of Science, 8 March str. 202, Ekaterinburg, RU-620144 Russia; e-mail: anton.g.shiryaev@gmail.com

Heikki Kotiranta, Finnish Environment Institute, Biodiversity Unit, P.O. Box 140, Helsinki, FI-00251 Finland; e-mail: heikki.kotiranta@ymparisto.fi

\section{Introduction}

Kotiranta \& Shiryaev (2015) published a list of aphyllophoroid fungi from Central Siberian State Biosphere Nature Reserve in river valley of Podkamennaja Tunguska (Stolbovoy field station). It is a tributary of the river Yenisei, about $100 \mathrm{~km}$ east of the study areas in this paper, which also belong (partly) to the Central Siberian State Biosphere Nature Reserve. Kotiranta \& Shiryaev (2015) also give a brief overview of the earlier Siberian and Russian Far East studies of aphyllophoroid fungi and this article completes the list of species of the Nature Reserve. When making comparisons with other studies in Westand East Siberia we compare the taxonomical and morphological parameters. The three main morphological groups are: corticioid, poroid and clavarioid fungi. This, fully artificial division is based on morphological characteristics only, but is practical when making comparisons between different basidiocarp forms in different climates.

\section{Material and methods}

The material was collected during the XXII International Trans-Siberian Mycological Expedition in the middle part of Yenisei river basin (Krasnoyarsk Kray, Turukhansk area) in August 2013. The material for this article was collected in three localities:

1) Surroundings of the village Bor (the Central Siberian Nature Reserve headquarter is in Bor), western side of Yenisei River (61³4`45.43” N, 9000`43.58” E). The collecting site is on the top of sandy dunes, with partly burned fairly young, poor Pinus sylvestris L. dominated heath forest site type with Cladina species. A few Alnus sibirica (Spach) Turcz. ex Kom. bushes lined the forest road. Here only two short collecting trips were made (16.8.2013 and 24.8.2013). Hill slopes are covered with 
mosses and grasses intermixed with Pinus sibirica Du Tour, Picea obovata Lebed., small Abies sibirica Lebed. trees and Juniperus communis L. bushes. River valleys and bog margins are wet with Sphagnum spp., Betula, Alnus, Salix, Padus, and some Athyrium filix-femina (L.) Roth. individuals.

2) The main collecting site was in the vicinity of the village Mirnoye $\left(62^{\circ} 17^{`} 44^{\prime \prime} \mathrm{N}, 8^{\circ} 00^{`} 49.5^{\prime}\right.$ ” E), on east side along the river Yenissei, about $80 \mathrm{~km}$ north of Bor. Here we collected for four days. The forests close to the village are relatively luxuriant. They were cut in 1940's during the Second World War, but after that they have been more or less intact (Fig. 1). The tree species here are: Abies sibirica, Alnus sibirica, Betula spp., Pinus sibirica, Populus tremula L., Padus avium Mill., Salix caprea L., Salix spp. plus small Juniperus communis shrubs. In boggy and moist habitats grew a few Matteuccia struthiopteris (L.) Tod. A short visit was made to the west shore of the river, just opposite to Mirnoye, where practically only Salix spp. bushes were growing.

3) When returning from Mirnoye to Bor, a short stop was made close to the village Lebed $\left(62^{\circ} 04^{`} 56.6^{\prime \prime} \mathrm{N}\right.$, $\left.89^{\circ} 11^{\prime} 13.8^{\prime \prime} \mathrm{E}\right)$. The forest was luxuriant, relatively old, but not in virgin state (cut stumps). The trees there were Abies sibirica, Alnus sibirica, Betula spp., Padus avium, Picea obovata, Pinus sibirica, Salix spp. and Sorbus sibirica Hedl. Some collections were made also from unidentified coniferous driftwood.

In central Yenisei region the average annual temperature is higher close to the Yenisei due to the warming effect of the valley, and the average is $-4.5^{\circ} \mathrm{C}$, falling eastwards to $-7.5^{\circ} \mathrm{C}$ and the mean temperature in January is $-23.6^{\circ}$ and in July $+18.2^{\circ} \mathrm{C}$, respectively (Harris et al. 2014). The average length of the growing period with temperature above $5^{\circ} \mathrm{C}$ is from 100 days in the north to 130 days in the south of the subzone, and above $10^{\circ} \mathrm{C}$, 65-70 and 90-100 days, respectively. The frost-free period varies from 60 in the north to 85 days in the south. Annual rainfall is $400-500 \mathrm{~mm}$ close to Yenisei River and the snow cover is $70-80 \mathrm{~cm}$ thick. The study area belongs to permafrost region where the ice depth reaches up to 25-50 m (Shherbina 2009).

Heterobasidioid and pleurotoid fungi are not treated in this study. Altogether 720 specimens of aphyllophoroid fungi were collected or only noted. Species like Fomes fomentarius, Fomitopsis pinicola, Phellinus tremulae, Piptoporus betulinus and Typhula uncialis, were mainly noted only (in list of species, sight). The material was identified in Ekaterinburg and Helsinki, and the specimens are deposited in the herbaria of Institute of Plant Animal Ecology, Ekaterinburg (SVER) and University of Helsinki $(\mathrm{H})$, or in the reference herbarium of Anton $\mathrm{G}$. Shiryaev $(A G S)$ and Heikki Kotiranta $(H K)$.

In the text the following abbreviations for the substrates are used: $\mathrm{Ab}=$ Abies sibirica, $\mathrm{Al}=$ Alnus sibirica, $\mathrm{B}=$ Betula spp., Jun = Juniperus communis, $\mathrm{L}=$ Larix sibirica, Pic $=$ Picea obovata, $\mathrm{Psi}=$ Pinus sibirica, Psy $=$ Pinus sylvestris, Pop $=$ Populus tremula, $\mathrm{Paa}=$ Padus avium, $\mathrm{S}=$ Salix spp., Scap $=$ Salix caprea $; \mathrm{Ssib}=$ Sorbus sibirica. CB means Cotton Blue, MLZ Melzers' reagent and $\mathrm{KOH} 3 \%$ potassium hydroxide.

The nomenclature mostly follows IndexFungorum (9 December 2014) with some modifications. Some of the material collected does not fit with any species known by us, and then a brief description is given. The species of the three basic morphological groups (corticioid, poroid and clavarioid) of aphyllophoroid fungi are arranged alphabetically regardless of their taxonomic possession. These groups have each their own chapter. The genus Steccherinum S.F. Gray is treated under poroid fungi, even if they are hydnoid, because their fruit bodies are macroscopically and especially microscopically closer to poroids (e.g., Antrodiella Ryvarden \& Johansen, Junghuhnia Corda emend. Ryvarden) than corticioids.

\section{List of species}

\section{Corticioid fungi}

Acanthobasidium lividocoeruleum (P. Karst.) Boid.: Bor, decorticated Psy, decay 4, diam. 18 $\mathrm{cm}$ (HK 26283), decorticated Psy, decay 3, diam. $28 \mathrm{~cm}(H K 26288)$.

Aleurodiscus amorphus (Pers. : Fr.) J. Schröt.: Mirnoye, Ab branch, decay 1, diam. $0.8 \mathrm{~cm}$ ( $H K$ 26399).

Amphinema byssoides (Pers. : Fr.) J. Erikss.: Lebed', decorticated $\mathrm{Al}$, decay 3, diam. $6 \mathrm{~cm}$ (HK 26427).

Amylocorticium subsulphureum (P. Karst.) Pouzar: Bor, decorticated Psy, decay 3, diam. $6.5 \mathrm{~cm}$ (HK 26284). We follow here the species concept of Eriksson \& Ryvarden (1973).

Aphanobasidium sp. (HK 26421): Lebed', corticated Ab branch, decay 1, diam. $1 \mathrm{~cm}$.

Fruit body resupinate, closely adnate, at first pruinose, later continuous, white, when dried cracking in irregular pieces $(\times 50)$, hyphal system monomitic, all hyphae clamped, thin-walled, often inflated, varying in width, mostly $1-3 \mu \mathrm{m}$, cystidia none, basidia very variable in shape, clavate or conical, often pleural, not rarely with protuberances in apical part, sometimes stalked (stalk up to $20 \mu \mathrm{m}$ long), 17-29(-34) $\times 7-11$ $\mu \mathrm{m}$, with four, sometimes two stout sterigmata, spores smooth, ellipsoid or somewhat ovoid, ventral side rarely concave, with slightly thickened walls, CB-, MLZ-, (8-)8.2-10(-10.2) $\times$ (4.7-)5-6.2(-7) $\mu \mathrm{m}, \mathrm{L}_{\mathrm{m}}=9.2 \mu \mathrm{m}, \mathrm{W}_{\mathrm{m}}=5.8 \mu \mathrm{m}$, $\mathrm{Q}=1.4-1.9, \mathrm{Q}_{\mathrm{m}}=1.6(\mathrm{n}=30)$.

Mature fruit body looks like Tubulicrinis subulatus except that there are no protruding cystidia under the lens $(\times 50)$. The spores are like in Den- 
drothele commixta (Höhn. \& Litsch.) J. Erikss. \& Ryvarden or the ovoid ones like in Hypochnicium bombycinum. Phlebiella rubi GrosseBrauckmann is basically similar to our specimen (see, Grosse-Brauckmann 2003, p. 97), but the stalked, pleural basidia which often bear different kinds of protuberances were not reported by her. The above mentioned species (except $T$. subulatus) have thickened cyanophilous spore walls, thus deviating from our specimen.

Athelia acrospora Jülich: Bor, fallen Psy close to the brook on wet shore ( $A G S$ 25822).

Athelia decipiens (Höhn. \& Litsch.) J. Erikss.: Mirnoye, decorticated B, decay 3, diam. $14 \mathrm{~cm}$ (HK 26340), corticated Al, decay 2, diam. $7 \mathrm{~cm}$ (HK 26370).

The specimen $H K 26370$ has "cystidia", which, however, in our opinion are results of prolonged growth of basidia. Basidia are stalked, often with internal repetition, which is typical for $A$. decipiens, and the spores are typical for A. decipiens.

Athelia epiphylla Pers.: Mirnoye, corticated Paa, decay 1, diam. $4 \mathrm{~cm}$ (HK 26393).

Athelopsis subinconspicua (Litsch.) Jülich (HK 26291): Bor, decorticated Psy, decay 3, diam. $28 \mathrm{~cm}$.

Hyphal system monomitic, all hyphae clamped, thin-walled, (1-)2-3 $\mu \mathrm{m}$ in diam., cystidia none, cystidioles not seen, basidia clavate, often with one constriction, stalked, (14-)16-20 $\times(4-) 4.5-5(-6) \mu \mathrm{m}$, with four, up to $5 \mu \mathrm{m}$ long sterigmata, spores ellipsoid to short-cylindrical, $5-6.5(-7.2) \times(2.7-) 3-4.2 \mu \mathrm{m}, \mathrm{L}_{\mathrm{m}}=5.7 \mu \mathrm{m}, \mathrm{W}_{\mathrm{m}}$ $=3.5 \mu \mathrm{m}, \mathrm{Q}=1.2-2.1, \mathrm{Q}_{\mathrm{m}}=1.6(\mathrm{n}=30)$. The spores are smaller than reported by Eriksson \& Ryvarden (1973, as Athelopsis hypochnoidea Jül.), but the size fits with those given by Kotiranta \& Saarenoksa (2005). The very narrow hyphae $(1 \mu \mathrm{m})$ are strange, but undoubtedly belong to this specimen.

Botryobasidium botryoideum (Overh.) Parmasto: Mirnoye, partly corticated B branch, decay 1, diam. $1 \mathrm{~cm}$ (HK 26330), corticated B branch, decay 1, diam. $2 \mathrm{~cm}(H K 26331)$, corticated B branch, decay 1, diam. $1.5 \mathrm{~cm}$ ( $H K 26335)$, corticated B branch, decay 4, diam. $0.6 \mathrm{~cm}(H K$ 26336a), partly corticated Jun, decay 2, diam. 2 $\mathrm{cm}$ (HK 26343), corticated Jun, decay 1, diam. $2 \mathrm{~cm}$ (HK 26344), corticated Psi branch, decay 1, diam. $1.5 \mathrm{~cm}$ (HK 26349), corticated Psi, decay 1, diam. $10 \mathrm{~cm}$ (HK 26353), decorticated Psi branch, decay 1, diam. $2.5 \mathrm{~cm}$ (HK 26357), decorticated Psi branch, decay 1, diam. $1.3 \mathrm{~cm}(H K$ 26358). The spores of all specimens are finely ornamented.

Botryobasidium botryosum (Bres.) J. Erikss.: Bor, decorticated Psy, decay 2, diam. $7 \mathrm{~cm}(H K$ 26274), decorticated Psy, decay 1, diam. $21 \mathrm{~cm}$ (HK 26277), decorticated Psy, decay 3, diam. 11 cm (HK 26281), decorticated Psy, decay 3, diam. $6.5 \mathrm{~cm}$ (HK 26285), decorticated Psy, decay 2, diam. $6 \mathrm{~cm}$ ( $H K$ 26299), decorticated Psy, decay 1, diam. $7 \mathrm{~cm}$ ( $H K$ 26301a), decorticated Psy roots, decay 4, diam. $20 \mathrm{~cm}$ ( $H K$ 26302), decorticated Psy, decay 3, diam. $12 \mathrm{~cm}$ (HK 26311).

Botryobasidium cf. candicans J. Erikss. (HK 26458): Bor, decorticated Psy, decay 1, diam. $4 \mathrm{~cm}$

Since the specimen was without an anamorph we are not quite sure about its identity.

Botryobasidium isabellinum (Fr.) D.P. Rogers: Lebed, decorticated coniferous driftwood, decay 1, diam. $17 \mathrm{~cm}$ (HK 26442).

Botryobasidium medium J. Erikss. (HK 26454): Bor, decorticated Psy, decay 2, diam. $3.5 \mathrm{~cm}$. The specimen was without an anamorph.

Botryobasidium subcoronatum (Höhn. \& Litsch.) Donk: Mirnoye, corticated Al, decay 3, diam. 5 cm (HK 26372).

Botryobasidium sp. (HK 26307): Bor, corticated Psy, decay 1, diam. $6.5 \mathrm{~cm}$. - Fig. 2

Fruit body resupinate, hypochnoid, pale greenish yellowish, margin distinct, all hyphae simple septate, smooth, basal ones 9-13 $\mu \mathrm{m}$ wide with thickened walls, contextual hyphae 7-9 $\mu \mathrm{m}$ wide, subhymenial hyphae $5-7.5 \mu \mathrm{m}$ in diam., cystidia relatively numerous, originate from bulbous cells, often with 1-3 septa, apically roundish, seldom widened into a up to $6 \mu \mathrm{m}$ wide head, (85-)105-165 × (7-)9-11 $\mu \mathrm{m}$, basidioles 


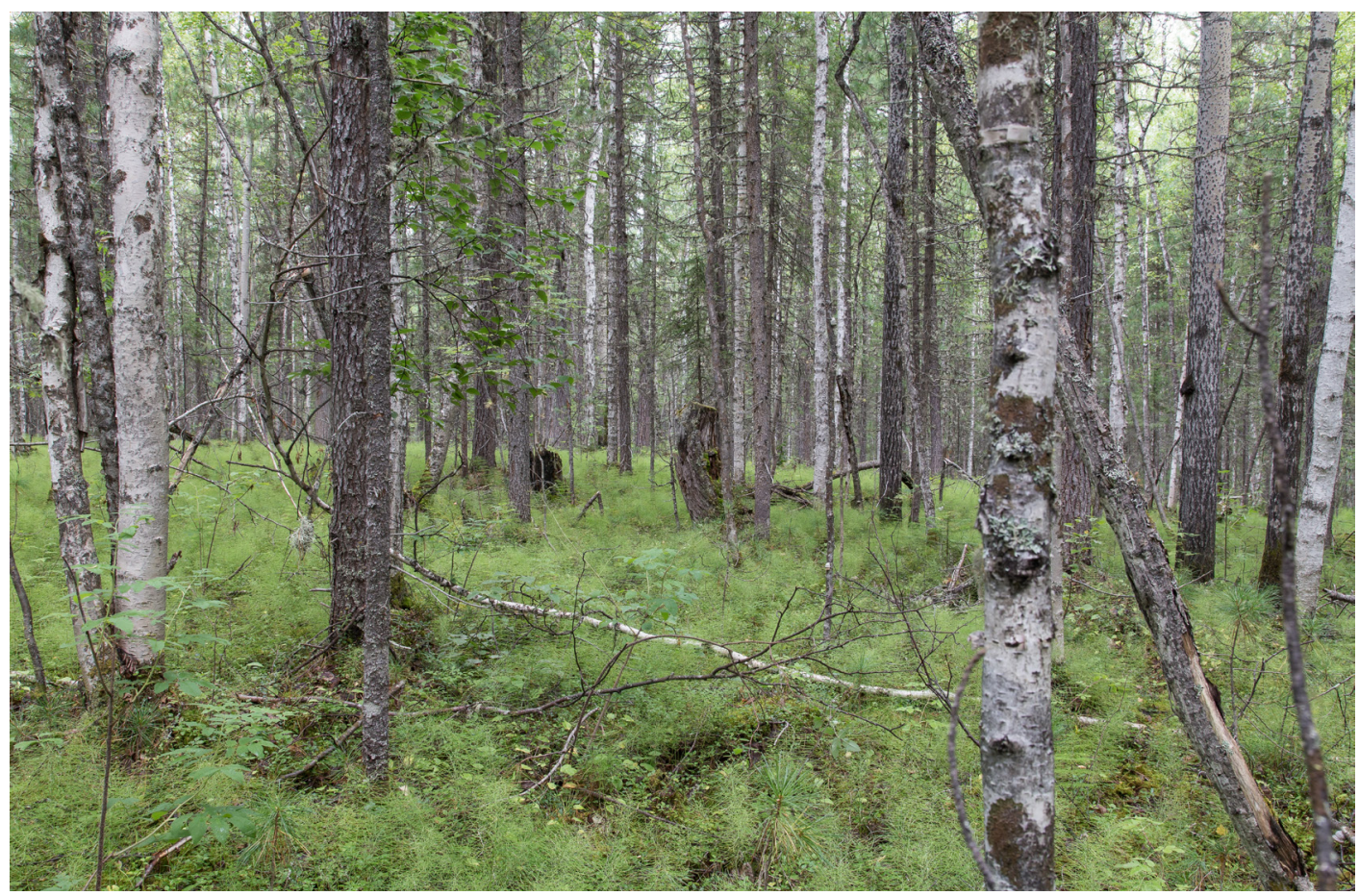

Fig. 1. Old mixed forest in Mirnoye.

subglobose, basidia subclavate, normally with one constriction, 12-17.5 $\times(9-) 10-12 \mu \mathrm{m}$, with $4-6$, up to $4.5 \mu \mathrm{m}$ long, quickly fading sterigmata, spores biapiculate, smooth, thin- to slightly thick-walled, cyanophilous (7.2-)8-10.3(-10.5) $\times(3.8-) 4-5(-5.2) \mu \mathrm{m}, \mathrm{L}_{\mathrm{m}}=9.1 \mu \mathrm{m}, \mathrm{W}_{\mathrm{m}}=4.3$ $\mu \mathrm{m}, \mathrm{Q}=1.7-2.4, \mathrm{Q}_{\mathrm{m}}=2.1(\mathrm{n}=30)$, often glued in pairs-tetrads

The shape and size of the spores is like an intermediate form of the spores of $B$. botryosum and B. obtusisporum J. Erikss. Other clamp less cystidiate Botryobasidium species have either smaller spores, like B. piliferum Boidin \& Gilles, or larger spores like $B$. digitatum (Rogers) $G$. Langer, or the cystidia do not have a bulbous base like $B$. tubulicystidatum $\mathrm{G}$. Langer, which moreover is described from Taiwan, from quite different climatic conditions (Langer, G. 1994). If our specimen is just $B$. botryosum with cystidia, at least the key of the genus Botryobasidium must be re-written.

Ceraceomyces serpens (Tode : Fr.) Ginns: Mirnoye, corticated Scap branch, decay 1, diam. $4 \mathrm{~cm}$ (HK 26397).
Chondrostereum purpureum (Pers. : Fr.) Pouzar: Mirnoye (west shore): corticated Scap, diam. 7 cm (AGS 25805).

Coniophora arida (Fr.) P. Karst.: Bor, decorticated Psy, decay 1, diam. $21 \mathrm{~cm}$ (HK 26276), corticated Psy, decay 3, diam. $12 \mathrm{~cm}$ (HK 26310).

Coniophora olivacea (Pers. : Fr.) P. Karst.: Mirnoye, decorticated Pic, decay 4, diam. $22 \mathrm{~cm}$ (HK 26381).

Corticium polygonioides P. Karst.: Mirnoye, decorticated B, decay 4, diam. $13 \mathrm{~cm}$ (HK 26342), corticated Scap, decay 1, diam. $7 \mathrm{~cm}$ (HK 26416).

Corticium roseum Pers. : Fr.: Mirnoye, corticated Pop branch, decay 1, diam. $1 \mathrm{~cm}$ (HK 26359), corticated S, decay 1, diam. $3 \mathrm{~cm}$ (HK 26362), corticated Scap, decay 1, diam. $9 \mathrm{~cm}$ (HK 26418), Scap (HK sight).

Crustoderma corneum (Bourdot \& Galzin) Nakasone: Bor, decorticated Psy, decay 3, diam. 6.5 cm (HK 26286). 

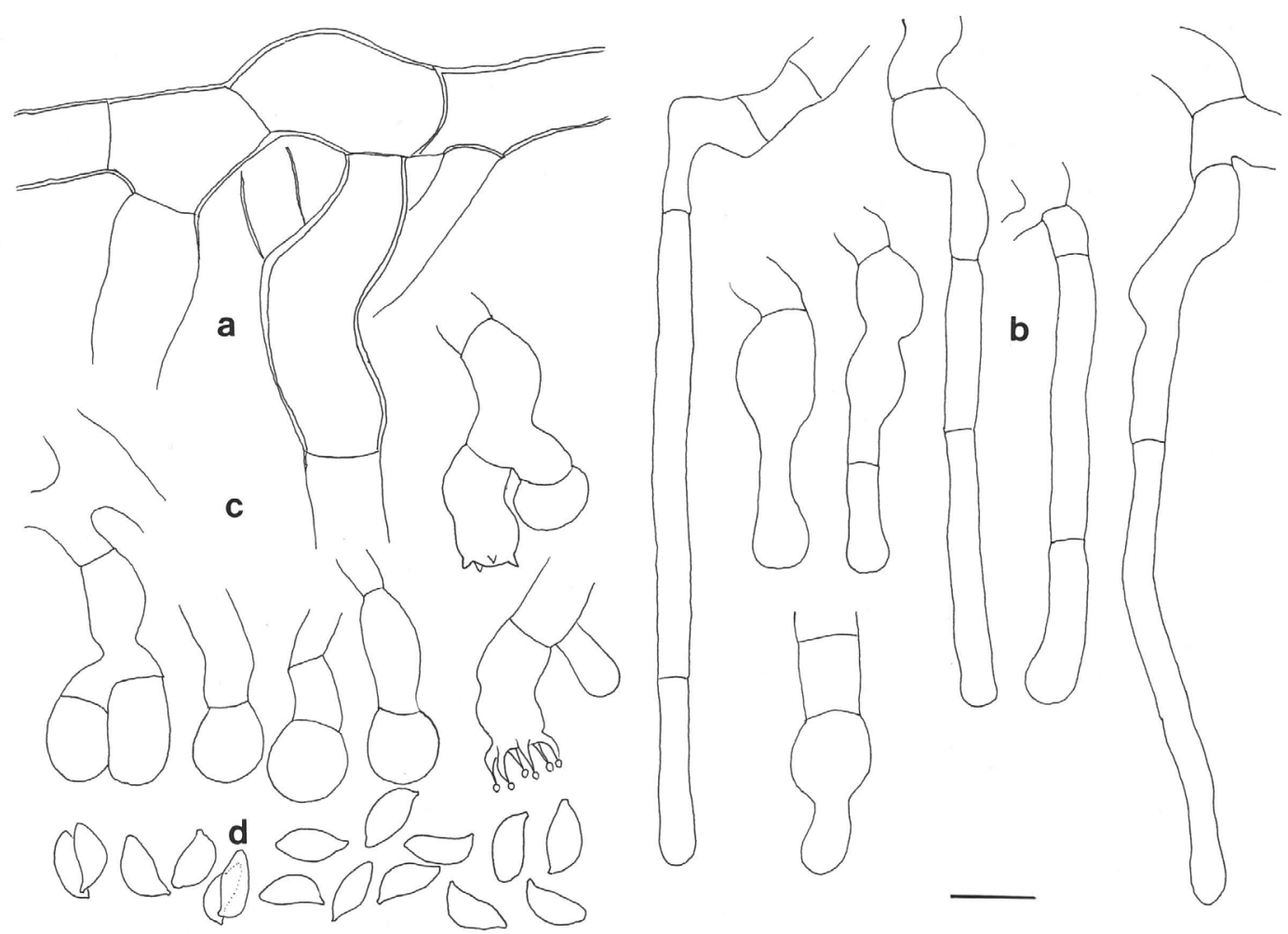

Fig. 2. Botryobasidium sp. (Kotiranta 26307). $a=$ subicular hyphae, $b=$ cystidia, $c=$ basidioles and basidia, $d=s p o r e s$. Scale bar $=10 \mu \mathrm{m}$.

Crustoderma dryinum (Berk. \& M.A. Curtis) Parmasto: Lebed', Pic branch (AGS 25865).

Cylindrobasidium evolvens (Fr. : Fr.) Jülich: Mirnoye (west shore), B trunk, diam. $6 \mathrm{~cm}$ (AGS 25906).

Cytidia salicina (Fr.) Burt: Mirnoye, corticated $\mathrm{S}$, decay 1, diam. $2 \mathrm{~cm}$ (HK 26363), corticated S, decay 1, diam. $7 \mathrm{~cm}$ (HK 26398).

Dacryobolus karstenii (Bres.) Oberw. ex Parmasto: Bor, decorticated Psy, decay 2, diam. 7 cm (HK 26304).

Fibricium rude (P. Karst.) Jülich: Mirnoye, corticated B branch, decay 1, diam. 2 cm (HK 26332), partly corticated Psi twigs, decay 2, diam. $0.2+$ $0.6 \mathrm{~cm}$ (HK 26348), corticated Psi branch, decay 2, diam. $0.7 \mathrm{~cm}$ (HK 26352), decorticated Psi, decay 1, diam. $4 \mathrm{~cm}$ (HK 26354), decorticated Psi branch, decay 1, diam. $2 \mathrm{~cm}$ (HK 26355), decorticated Psi branch, decay 1, diam. $2.5 \mathrm{~cm}(H K$ 26356).

Globulicium hiemale (Laurila) Hjortstam: Mirnoye, Psy branch (AGS 25839).

Hymenochaete tabacina (Sowerby) Löv.: Mirnoye, corticated Al, decay 4, diam. $4 \mathrm{~cm}(H K$ 26327), corticated Al, decay 2, diam. $6 \mathrm{~cm}$ (HK 26424), Lebed', corticated Pic, decay 2, diam. $1.5 \mathrm{~cm}$ (HK 26435).

Hyphoderma crassescens Laurila ex. K.H. Larss. nom. prov. (HK 26280): Bor, decorticated Psy, decay 3 , diam. $11 \mathrm{~cm}$.

Hyphal system monomitic, hyphae clamped, in subiculum thick-walled (up to $1 \mu \mathrm{m}$ ), $4-5 \mu \mathrm{m}$ wide, swelling in $\mathrm{KOH}$, others thin-walled, acy- 
anophilous without crystals in $\mathrm{CB}$, but encrusted in MLZ, cystidia none, but fusoid cystidioles common, not projecting above the basidia, 19$26 \times 3.5-4(-5) \mu \mathrm{m}$, basidia subclavate or utriform, often with an appendix, 24-34 × 5-6(-7) $\mu \mathrm{m}$, spores ellipsoid, $(5.8-) 6-7(-7.2) \times(3-) 3.2-$ $4.1(-4.5) \mu \mathrm{m}, \mathrm{L}_{\mathrm{m}}=6.5 \mu \mathrm{m}, \mathrm{W}_{\mathrm{m}}=3.7 \mu \mathrm{m}, \mathrm{Q}=$ $1.5-2.3, \mathrm{Q}_{\mathrm{m}}=1.8$, with a very small apiculus, thin-walled, CB-, MLZ- $(\mathrm{n}=30)$.

This species is not rare in Finland and Russia in burned pine forests, where it often grows on charred pines. A somewhat similar species is Hyphoderma sibiricum (Parmasto) J. Erikss. \& Strid, but it is much thinner, the spores have a relatively large apiculus, and it never has basidia with an appendix. For the identification the best characteristics are the basidia which often have an appendix - a feature, which is lacking from other Hyphoderma species.

Hyphoderma nemorale K.H. Larss. (HK 26347): Mirnoye, corticated Pic twig, decay 1, diam. 0.4 $\mathrm{cm}$.

$H$. nemorale mostly grows on deciduous trees (Larsson 1998), and we do not know other specimens collected from coniferous wood.

Hyphoderma setigerum (Fr.) Donk s. lato: Bor, decorticated Psy, decay 1, diam. $4 \mathrm{~cm}$ (HK 26308), Mirnoye, corticated B, decay 2, diam. 2 $\mathrm{cm}(H K 26337)$, corticated B, decay 1, diam. 1.5 cm (HK 26341), dead, erect corticated Al, decay 2, diam. $5 \mathrm{~cm}$ (HK 26365), Lebed', corticated Al, decay 2 , diam. $4 \mathrm{~cm}(H K 26422)$, corticated Al, decay 2, diam. $8 \mathrm{~cm}$ (HK 26431).

Hyphodontia alutacea (Fr.) J. Erikss.: Mirnoye, Pic trunk (AGS 25891).

Hyphodontia aspera (Fr.) J. Erikss.: Bor, fallen Psy trunk, under the bark (AGS 25927).

Hyphodontia barba-jovis (Bull. : Fr.) J. Erikss.: Mirnoye, corticated B, decay 3, diam. $4 \mathrm{~cm}(H K$ 26403).

Hyphodontia breviseta (P. Karst.) J. Erikss.: Bor, decorticated Psy, decay 4, diam. $11 \mathrm{~cm}(H K$ 26452), decorticated Psy, decay 4, diam. $20 \mathrm{~cm}$ (HK 26462b).
Hyphodontia crustosa (Pers.) J. Erikss.: Mirnoye, decorticated Psy, diam. $13 \mathrm{~cm}$ (AGS 25863).

Hyphodontia quercina (Pers. : Fr.) J. Erikss.: Mirnoye, corticated Al, decay 3, diam. $2 \mathrm{~cm}(H K$ 26314), corticated Al branch, decay 3, diam. 1.5 cm (HK 26316), corticated Al, decay 3, diam. 2 $\mathrm{cm}$ (HK 26322), co Al branch, decay 4, diam. 1.5 $\mathrm{cm}$ (HK 26400).

The specimen $H K 26314$ deviates a bit from the others we collected. Some subicular hyphae bear richly branched, bush-like side-branches, and some cystidioles, especially the subulate ones have clearly oily contents, like gloeocystidia, 25-30 × 4-5 $\mu \mathrm{m}$, basidia, cylindrical, 23-29 $\times 4-5.5 \mu \mathrm{m}$, with one constriction, spores cylindrical, thin-walled, $(6.2-) 6.5-8.1 \times 2.1-3.1 \mu \mathrm{m}$, $\mathrm{L}_{\mathrm{m}}=7.1 \mu \mathrm{m}, \mathrm{W}_{\mathrm{m}}=2.7 \mu \mathrm{m}, \mathrm{Q}=2.3-3.3, \mathrm{Q}_{\mathrm{m}}=$ $2.7(n=30)$.

Hyphodontia cf. spathulata (Schrad.) Parmasto (HK 26350): Mirnoye, corticated Psi branch, decay 2, diam. $1.5 \mathrm{~cm}$.

Fruit body resupinate, adnate, creamish, hymenium porose-reticulate between the mostly cylindrical, seldom flattened up to $1 \mathrm{~mm}$ long aculei, all hyphae clamped, tramal hyphae with slightly thickened walls (up to $0.4 \mu \mathrm{m}$ ), 2-3(-5) $\mu \mathrm{m}$ in diam., hyphae of dissepiment edge often glued together, 2-3 $\mu \mathrm{m}$ wide, cystidia of three kinds: a) sword-shaped, (32-)36-49 × (3-)3.5$4.5 \mu \mathrm{m}, \mathrm{b})$ capitate, slightly thick-walled, 26-31 $\times 4-5 \mu \mathrm{m}$, and c) gloeocystidia, very pale yellowish in $\mathrm{KOH}$, strongly cyanophilous (contents), apically obtuse or sharp-pointed, sometimes moniliform, and apically branching, relatively often with appendix-like protuberances, (15)20-59 × 4-7 $\mu \mathrm{m}$, basidia cylindrical, 20-24 $\times$ 4-5.5 $\mu \mathrm{m}$, with four sterigmata, spores ellipsoid or broadly ellipsoid, 4.5-5(-5.2) $\times(3.5-) 3.7-4.2$ $\mu \mathrm{m}, \mathrm{L}_{\mathrm{m}}=4.8 \mu \mathrm{m}, \mathrm{W}_{\mathrm{m}}=3.9 \mu \mathrm{m}, \mathrm{Q}=1.1-1.4, \mathrm{Q}_{\mathrm{m}}$ $=1.2(\mathrm{n}=30)$.

The capitate cystidia, as well as the gloeocystidia fit with the concept of $H$. spathulata, except that the gloeocystidia often bear appendix-like protuberances. Also the spores fit with $H$. spathulata, but we have not seen sword-like cystidia before in this taxon.

Hyphodontia subalutacea (P. Karst.) J. Erikss.: Mirnoye, decorticated Pic, diam. $11 \mathrm{~cm}$ ( $A G S$ 25947). 
Hyphodontia sp. (HK 26439): Lebed', decorticated Pic, decay 2, diam. $13 \mathrm{~cm}$.

Fruit body resupinate, adnate, odontioid with up to $0.9 \mathrm{~mm}$ long cylindrical, sometimes denticulate creamish aculei, hymenium between the aculei porose-reticulate, white, all hyphae clamped, subicular ones with thickened walls, 3-4 $\mu \mathrm{m}$ wide, tramal 2-3 $\mu \mathrm{m}$ in diam. with small crystals, no capitate cystidia or gloeocystidia, hymenial cystidioles very thin-walled, spear-shaped, rarely with slightly widened apex, 35-45 $\times 4-5 \mu \mathrm{m}$, similar to the cystidioles in apical apices of the aculei, basidia cylindrical or subcylindrical, normally with two constrictions, (20-)24-30 × 4-4.5 $\mu \mathrm{m}$, with four, up to $4 \mu \mathrm{m}$ long sterigmata, spores ellipsoid or broadly ellipsoid, thin-walled, 4.6-5.2 $\times 3.7-4.2 \mu \mathrm{m}, \mathrm{L}_{\mathrm{m}}=4.9$ $\mu \mathrm{m}, \mathrm{W}_{\mathrm{m}}=3.9 \mu \mathrm{m}, \mathrm{Q}=1.1-1.4, \mathrm{Q}_{\mathrm{m}}=1.3(\mathrm{n}=30)$.

In outer appearance the fruit body is very similar to Hyphodontia cf. spathulata (HK 26350) like the shape and size of the spores. However, this specimen has only spear-shaped cystidioles similar to those in Hyphodontia nudiseta (Warcup \& P.H.B. Talbot) Hjortstam \& Ryvarden, drawn by E. Langer (1994, p. 167). Also the spores fit relatively well in that species, even if they here are more roundish. According to our experience the fruit body of $H$. nudiseta is softer, normally white and the aculei are much shorter.

Hypochnicium bombycinum (Sommerf.) J. Erikss.: Mirnoye, corticated S, decay 1, diam. 4 cm (HK 26364), corticated Al, decay 2, diam. 9 cm (HK 26368).

Hypochnicium punctulatum (Cooke) J. Erikss.: Bor, fallen Psi in river valley (AGS 25916). We follow here the species concept of Eriksson and Ryvarden (1976).

Intextomyces contiquus (P. Karst.) J. Erikss. \& Ryvarden: Mirnoye, corticated Al, decay 4, diam. $4.5 \mathrm{~cm}$ (HK 26325), corticated Paa, decay 3, diam. $3 \mathrm{~cm}$ (HK 26390), corticated Scap snag, decay 2, diam. $10 \mathrm{~cm}$ (HK 26413), living, corticated Scap, diam. $13 \mathrm{~cm}$ (HK 26415), corticated Scap, decay 2, diam. 16 cm (HK 26419).

Laurilia sulcata (Burt) Pouzar: Lebed': corticated L, diam. $18 \mathrm{~cm}$ ( $A G S$ 25881).
Laxitextum bicolor (Pers. : Fr.) Lenzt: Mirnoye, corticated Al, decay 3, diam. $6 \mathrm{~cm}$ (HK 26367).

Leptosporomyces galzinii (Bourdot) Jülich: Bor, decorticated Psy, decay 1, diam. $21 \mathrm{~cm}$ (HK 26278), decorticated Psy branch, decay 1, diam. $3 \mathrm{~cm}$ (HK 26449).

Leucogyrophana mollusca (Fr.) Pouzar: Lebed, decorticated Psi driftwood, decay 2, diam. $21 \mathrm{~cm}$ (HK 26441).

Mycoacia fuscoatra (Fr. : Fr.) Donk: Bor, B trunk, diam. $15 \mathrm{~cm}$ (AGS 25816).

Peniophora aurantiaca (Bres.) Höhn. \& Litsch.: Bor, corticated Al, decay 2, diam. $5 \mathrm{~cm}(H K$ 26270).

Peniophora erikssonii Boidin: Lebed', corticated Al, decay 2, diam. $4 \mathrm{~cm}$ (HK 26423).

Peniophora pithya (Pers.) J. Erikss.: Mirnoye, Pic branch, diam. $4 \mathrm{~cm}$ (AGS 25968).

Peniophora rufa (Fr.) Boidin: Mirnoye, corticated Pop, decay 1, diam. $20 \mathrm{~cm}$ (HK 26389).

Peniophorella praetermissa (P. Karst.) K.H. Larss.: Mirnoye, corticated Psi branch, decay 2, diam. $1.5 \mathrm{~cm}$ (HK 26351).

Phanerochaete sanguinea (Fr.) Pouzar: Mirnoye, fallen Psi branch (AGS 25934).

Phanerochaete sordida (P. Karst.) J. Erikss. \& Ryvarden s. lato: Bor, decorticated Psy branch, decay 2, diam. $4 \mathrm{~cm}$ (HK 26461), Mirnoye, corticated $\mathrm{Al}$, decay 3, diam. $2 \mathrm{~cm}$ (HK 26324), co B twigs, decay 4, diam. $0.2 \mathrm{~cm}+0.4 \mathrm{~cm}(H K$ $26328)$, corticated B branch, decay 4 , diam. $6 \mathrm{~cm}$ (HK 26329).

Phanerochaete velutina (DC. : Fr.) P. Karst.: Mirnoye, corticated $\mathrm{Al}$ decay 3, diam. $5 \mathrm{~cm}$ (HK 26313), decorticated $\mathrm{Al}$, decay 3 , diam. $8 \mathrm{~cm}$ (HK 26318), partly corticated Al, decay 3, diam. $7 \mathrm{~cm}$ (HK 26319), corticated Al, decay 4, diam. 6 cm (HK 26326), corticated Pop branch, decay 2, diam. $1 \mathrm{~cm}$ (HK 26360). 
Phlebia centrifuga P. Karst.: Lebed', decorticated Pic, decay 3, diam. $13 \mathrm{~cm}$ (HK 26436).

Phlebia lilascens (Bourdot) J. Erikss. \& Hjortstam: Mirnoye, decorticated Pic, decay 3, diam. 8 cm (HK 26345).

Phlebia nitidula (P. Karst.) Ryvarden: Mirnoye, corticated Scap, decay 1, diam. $4.5 \mathrm{~cm}$ (HK 26361).

Phlebia segregata (Bourdot \& Galzin) Parmasto: Bor, coniferous board, decay 2, diam. $10 \times 1.5$ cm (HK 26447).

Phlebia tremellosa (Schrad. : Fr.) Nakasone: Mirnoye (west shore): corticated Al, diam. $5 \mathrm{~cm}$ (AGS 25899).

Phlebiella pseudotsugae (Burt) K.H. Larss. \& Hjortstam: Bor, fallen Psy, moist river valley (AGS 25850).

Phlebiella sulphurea (Pers. : Fr.) Ginns \& M.N.L. Lefebvre: Mirnoye, Pic trunk and mosses on bog (AGS 25954).

Piloderma byssinum (P. Karst.) Jülich: Bor, decorticated Psy branch on slope (AGS 25835).

Piloderma sp. (HK 26426): Lebed', decorticated Al, decay 3, diam. $6 \mathrm{~cm}$.

Fruit body like in $P$. byssinum, without rhizomorphs, all hyphae simple septate, in narrow subicular strands $1.5-2 \mu \mathrm{m}$ in diam., with rodlike crystals, subhymenial hyphae thin-walled, 2-3.5 $\mu \mathrm{m}$ wide, cystidia none, basidia clavate, stalked, $15-19.5(-22) \times 4-5 \mu \mathrm{m}$, with two, relatively stout, up to $5.5 \mu \mathrm{m}$ long sterigmata, spores ellipsoid or broadly ellipsoid, thick-walled, strongly cyanophilous, MLZ-, 4-5(-5.8) × (3.3)3.5-4.1 $\mu \mathrm{m}, \mathrm{L}_{\mathrm{m}}=4.5 \mu \mathrm{m}, \mathrm{W}_{\mathrm{m}}=3.8 \mu \mathrm{m}, \mathrm{Q}=$ $1.1-1.3, \mathrm{Q}_{\mathrm{m}}=1.2(\mathrm{n}=30)$.

Piloderma byssinum sometimes has two-spored basidia, but the spores are smaller and differently shaped. Piloderma lanatum var. bisporum (Parmasto) J. Erikss. \& Hjortstam has also constantly bi-sterigmatic basidia, but the size and shape of the spores differ (Eriksson et al. 1981) from our specimen. We have another specimen of the taxon described above: Russia, Krasnoyarsk Kray,
Abakan S, Erkagi Nature Park, Tushkanchik River Visit Centre $\left(52^{\circ} 45^{\prime} \mathrm{N}, 93^{\circ} 21^{\prime} \mathrm{E}\right.$, alt. 1110 $\mathrm{m}$ a.s.1.), old-growth forest, decorticated, mosscovered Picea obovata, decay 3, diam. $40 \mathrm{~cm}$ (16.8.2011, HK 23101).

Plicatura nivea (Sommerf. : Fr.) P. Karst.: Mirnoye, corticated Al, decay 2, diam. $7 \mathrm{~cm}(H K$ 26369), corticated Al, decay 3, diam. $6 \mathrm{~cm}+3$ (HK sight).

Resinicium furfuraceum (Bres.) Parmasto: Bor, decorticated Psy, decay 4, diam. $18 \mathrm{~cm}$ (HK 26282), decorticated Psy, decay 1, diam. $7 \mathrm{~cm}$ (HK 26300), decorticated Psy, decay 3, diam. 19 cm (HK 26453).

Scytinostroma galactinum (Fr.) Donk: Mirnoye, corticated B, decay 4, diam. $10 \mathrm{~cm}$ (HK 26334).

Sistotrema brinkmannii (Bres.) J. Erikss.: Mirnoye, corticated Paa, decay 1, diam. $4 \mathrm{~cm}$ (HK 26392).

Sistotrema oblongisporum M.P. Christ. \& Hauerslev: Bor, corticated Al branch, decay 1, diam. $1.2 \mathrm{~cm}$ (HK 26268).

Sistotrema octosporum (J. Schröt. ex Höhn. \& Litsch.) Hallenb.: Mirnoye, decorticated Al, decay 2, diam. $6 \mathrm{~cm}$ (HK 26375). Relatively poor material, and spores a bit too wide.

Sistotremastrum niveocremeum (Höhn. \& Litsch.) J. Erikss.: Bor, corticated Al, decay 2, diam. $5 \mathrm{~cm}$ (HK 26267).

Sistotremastrum suecicum Litsch. ex J. Erikss.: Bor, decorticated Psy, decay 2, diam. $8 \mathrm{~cm}$ (HK 26287), coniferous board, decay 2 , diam. $10 \times$ $1.5 \mathrm{~cm}$ (HK 26446), decorticated Psy, decay 4, diam. $13 \mathrm{~cm}$ (HK 26451).

Stereum hirsutum (Willd. : Fr.) Gray: Mirnoye (west shore), corticated S branch (AGS 25909).

Stereum sanguinolentum (Alb. \& Schwein. : Fr.) Fr.: Lebed', corticated Pic, diam. $18 \mathrm{~cm}$ (AGS 25937). 
Subulicystidium longisporum (Pat.) Parmasto: Mirnoye, decorticated Jun, decay 4, diam. $1 \mathrm{~cm}$ (HK 26406).

Tomentella atramentaria Rostr.: Bor, fallen Psy branch in mosses in wet habitat (AGS 25976).

Tomentella bryophila (Pers.) M.J. Larsen: Lebed', fallen Ssib branch, diam. $3 \mathrm{~cm}$ (AGS 25873).

Tomentella ellisii (Sacc.) Jülich \& Stalpers: Mirnoye, decorticated B branch, diam. $4 \mathrm{~cm}$ (AGS 25802).

Tomentella fuscocinerea (Pers. : Fr.) Donk: Bor, Psy trunk in wet habitat, diam. $9 \mathrm{~cm}(A G S$ 25845).

Tomentella radiosa (P. Karst.) Rick: Mirnoye, decorticated Pic trunk, diam. $11 \mathrm{~cm}(A G S 25890)$.

Tubulicrinis borealis J. Erikss.: Bor, decorticated Psy, decay 2, diam. $3.5 \mathrm{~cm}$ (HK 26455).

Tubulicrinis calothrix (Pat.) Donk: Bor, decorticated Psy branch, decay 1, diam. $4 \mathrm{~cm}$ (HK 26460), Lebed', decorticated Pic, decay 3, diam. $13 \mathrm{~cm}$ (HK 26437).

Tubulicrinis chaetophorus (Höhn.) Donk: Bor, decorticated Psy, decay 3, diam. $28 \mathrm{~cm}(H K$ 26289).

Tubulicrinis glebulosus (Bres.) Donk: Bor, partly corticated Al, decay 2, diam. $3 \mathrm{~cm}$ (HK 26445), partly corticated Psy, decay 2, diam. $3.5 \mathrm{~cm}(H K$ 26456), decorticated Psy, decay 1, diam. $4 \mathrm{~cm}$ (HK 26457).

Tubulicrinis medius (Bourdot \& Galzin) Oberw.: Bor, decorticated Psy, decay 3, diam. $23 \mathrm{~cm}$ (HK 26297), decorticated Psy, decay 1, diam. $7 \mathrm{~cm}$ (HK 26301b), decorticated Psy roots, decay 4, diam. $20 \mathrm{~cm}$ (HK 26303).

Tubulicrinis sororius (Bourdot \& Galzin) Oberw.: Lebed', corticated Ab branch, decay 1, diam. $1 \mathrm{~cm}$ (HK 26420).

Tubulicrinis strangulatus K.H. Larss. \& Hjortstam: Bor, partly corticated Psy, decay 3, diam. 12 cm (HK 26312).
Tubulicrinis subulatus (Bourdot \& Galzin) Donk: Mirnoye, decorticated Psy, diam. $13 \mathrm{~cm}$ (AGS 25965).

Tylospora asterophora (Bonord.) Donk: Bor, partly decorticated Psy, diam. $9 \mathrm{~cm}$, in wet habitat (AGS 25922).

Veluticeps abietina (Pers. Fr.) Hjortstam \& Telleria: Lebed', fallen, partly decorticated Pic, diam. $10 \mathrm{~cm}$ ( $A G S$ 25857).

\section{Poroid fungi}

Antrodia albobrunnea (Romell) Ryvarden: Lebed', fallen, corticated Pic, diam. $15 \mathrm{~cm}$ (AGS 25913).

Antrodia serialis (Fr.) Donk: Lebed', decorticated Pic, decay 3, diam. $13 \mathrm{~cm}$ (HK 26438).

Antrodia sinuosa (Fr.) P. Karst.: Bor, decorticated Psy, decay 2, diam. $21 \mathrm{~cm}$ (HK 26292).

Antrodia xantha (Fr. : Fr.) Ryvarden: Bor, decorticated Psy, decay 2, diam. 7 cm (HK 26273), decorticated Psy branch, decay 3, diam. $6 \mathrm{~cm}(H K$ sight), decorticated Psy branch, decay 1, diam. $4 \mathrm{~cm}$ (HK 26459), Mirnoye, decorticated Scap snag, decay 2, diam. $10 \mathrm{~cm}$ ( $H K$ 26412).

Antrodiella pallescens (Pilát) Niemelä \& Miettinen: Mirnoye, corticated Al, decay 3, diam. $4 \mathrm{~cm}$ (HK 26321).

Bjerkandera adusta (Willd. : Fr.) P. Karst.: Mirnoye, B (HK sight), Pop (HK sight).

Cerrena unicolor (Bull. : Fr.) Murrill: Bor, corticated $\mathrm{Al}$, decay 2, diam. $5 \mathrm{~cm}$ (HK 26266), B stump, decay 2, diam. $18 \mathrm{~cm}$ (HK 26462), B (HK sight).

Cinereomyces lindbladii (Berk.) Jülich: Bor, decorticated Psy, decay 3, diam. $11 \mathrm{~cm}(H K$ 26280), decorticated Psy, decay 2, diam. $21 \mathrm{~cm}$ (HK 26293).

Coltricia perennis (L. : Fr.) Murrill: Bor, on sandy soil (AGS 25931); Mirnoye, on soil (AGS 25811). 


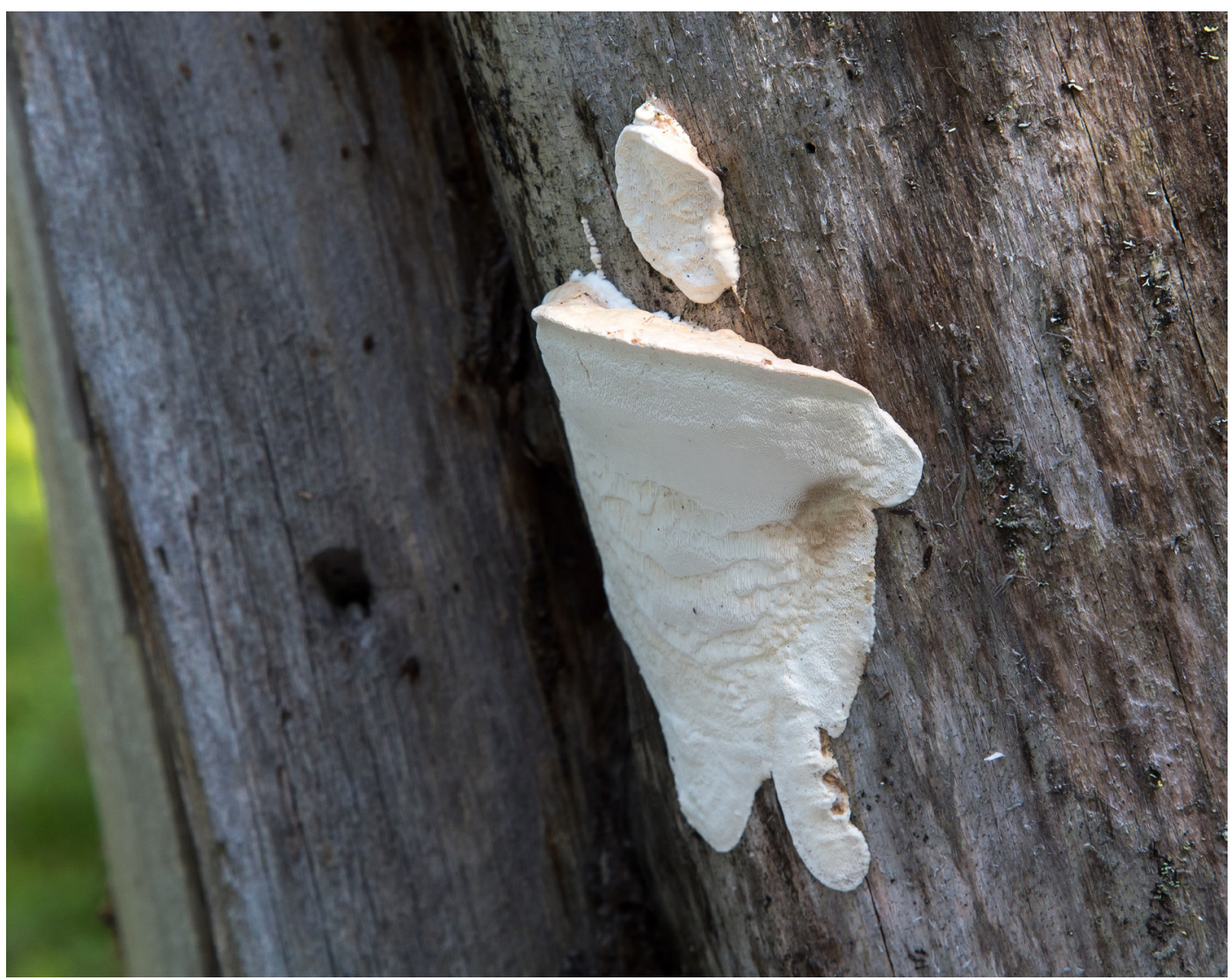

Fig. 3. Dichomitus squalens on Pinus sylvestris in Mirnoye.

Daedaleopsis confragosa (Bolton : Fr.) Schröt.: Mirnoye, corticated Scap, decay 1, diam. $12 \mathrm{~cm}$ $(H K 26396+$ sight $)$.

Daedaleopsis tricolor (Pers.) Bondartsev \& Singer: Mirnoye, corticated Al, decay 3, diam. 6 $\mathrm{cm}$ (HK 26320), corticated Al, decay 3, diam. 4 cm (HK 26373), corticated B (HK sight), Lebed', corticated B, decay 2, diam. $5 \mathrm{~cm}$ (HK 26432).

Datronia mollis (Sommerf.) Donk: Mirnoye, corticated Paa, diam. $5 \mathrm{~cm}$ ( $A G S$ 25886).

Dichomitus squalens (P. Karst.) D.A. Reid - Fig. 3

Bor, decorticated Psy, decay 1, diam. $21 \mathrm{~cm}(H K$ 26275), corticated Psy, decay 4, diam. $18 \mathrm{~cm}$.
(HK 26305), corticated Psy, decay 3, diam. 16 cm (HK 26306), Mirnoye, decorticated, dead, erect Psi, decay 1, diam. $53 \mathrm{~cm}$ (HK 26407).

Fomes fomentarius (L. : Fr.) Fr.: Mirnoye, corticated $\mathrm{B} \times 20$ (HK sight), Lebed', corticated $\mathrm{B}$, decay 3, diam. $5 \mathrm{~cm}$ (HK sight).

Fomitopsis cajanderi (P. Karst.) Kotl. \& Pouzar: Lebed, decorticated coniferous driftwood, decay 2, diam. $19 \mathrm{~cm}$ (HK 26443).

Fomitopsis pinicola (Sw. : Fr.) P. Karst.: Bor, decorticated Psy, decay 1, diam. $21 \mathrm{~cm}(H K$ sight $)$, Mirnoye, corticated Al, decay 2, diam. $7 \mathrm{~cm}(H K$ 26366), corticated Pic, decay 2, diam. $8 \mathrm{~cm}$ (HK sight $), \mathrm{Al} \times 4(H K$ sight $), \mathrm{B} \times 3($ HK sight $), \mathrm{Psi}$ (HK sight). 


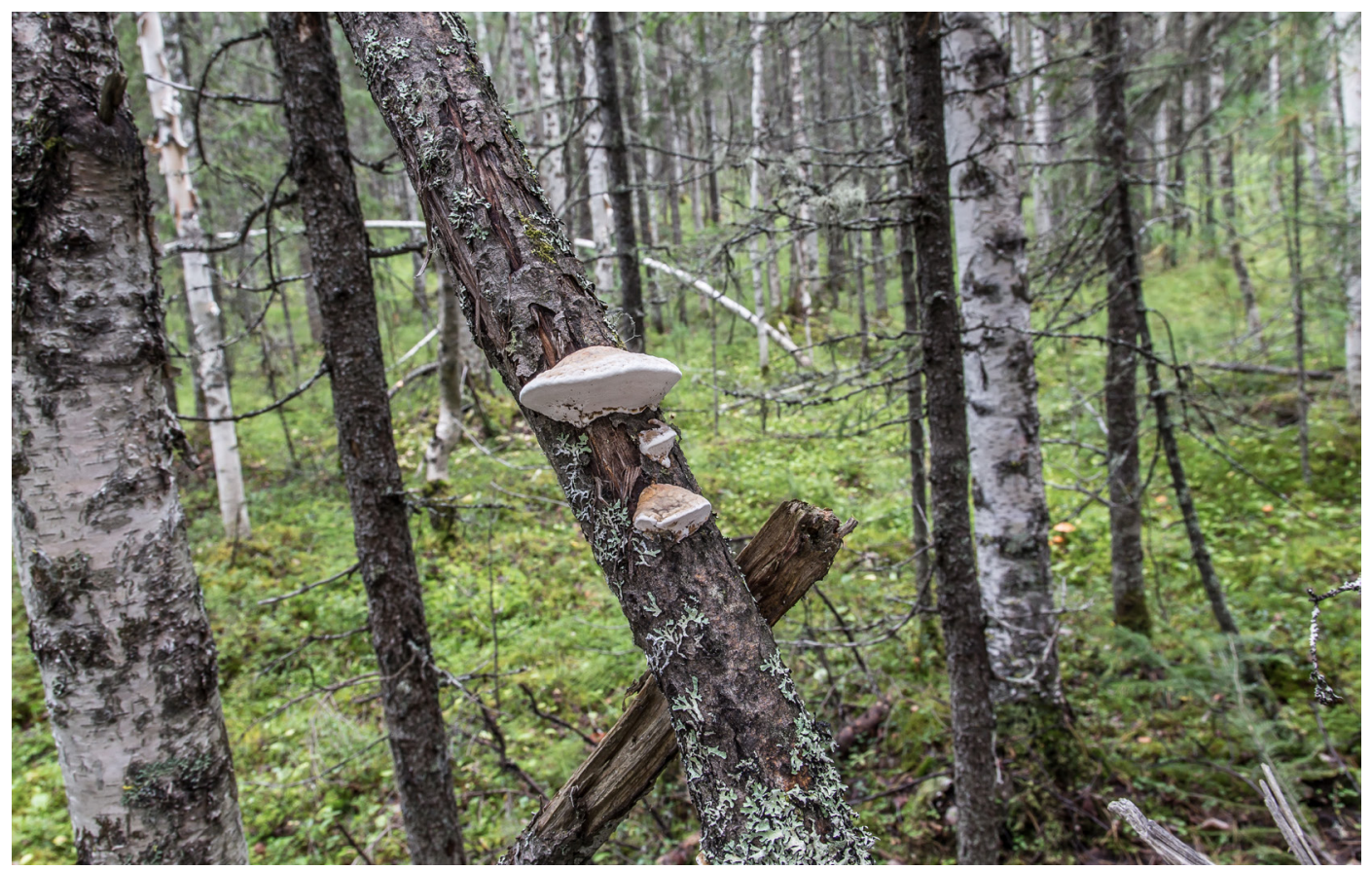

Fig. 4. Haploporus odorus on Salix caprea in Mirnoye.

Fomitopsis rosea (Alb. \& Schwein. : Fr.) P. Karst.: Lebed', decorticated, Pic, decay 3, diam. $13 \mathrm{~cm}$ (dead fruit body, $H K$ sight).

Ganoderma applanatum (Pers.) Pat.: Mirnoye, decorticated Al, decay 2, diam. $7 \mathrm{~cm}$ (HK 26376).

Gelatoporia subvermispora (Pilát) Niemelä: Bor, decorticated Psy, decay 4, diam. $13 \mathrm{~cm}(H K$ 26450).

Gloeophyllum sepiarium (Wulfen : Fr.) P. Karst.: Mirnoye, decorticated Psi, decay 2, diam. $50 \mathrm{~cm}$ (HK 26383), Lebed', decorticated coniferous driftwood, decay 2, diam. $17 \mathrm{~cm}$ (HK 26444).

Hapalopilus rutilans (Pers. : Fr.) P. Karst.: Mirnoye, B (dead fruit body, $H K$ sight).

Haploporus odorus (Sommerf.) Bondartsev \& Singer $\quad-$ Fig. 4

Mirnoye, corticated Scap, decay 2, diam. $9 \mathrm{~cm}$ (HK 26394), dead, erect, corticated Scap, decay
2, diam. $9 \mathrm{~cm}$ ( $H K$ 26395), fallen, corticated Scap, decay 3, diam. $9 \mathrm{~cm}$ ( $H K$ 26409), dead, erect, corticated Scap, decay 3, diam. $6 \mathrm{~cm}(H K$ 26410), living Scap, diam. $13 \mathrm{~cm}$ (HK 26414).

Inonotus obliquus (Pers. : Fr.) Pilát: Mirnoye, B snag $\times 2(H K$ sight $)$.

Inonotus radiatus (Sowerby : Fr.) P. Karst.: Mirnoye, decorticated Al, decay 3, diam. $8 \mathrm{~cm}$ (HK 26317), corticated $\mathrm{Al}$, decay 3, diam. 5 $\mathrm{cm}(H K 26371)$, corticated $\mathrm{Al} \times 2(H K$ sight $)$, Lebed', corticated Al, decay 2 , diam. $8 \mathrm{~cm}(H K$ 26430).

Inonotus rheades (Pers.) Bondartsev \& Singer: Mirnoye, corticated Pop (HK 26388).

Irpex lacteus (Fr. : Fr.) Fr.: Mirnoye (west shore), corticated S, diam. $2 \mathrm{~cm}$ (AGS 25826).

Laetiporus sulphureus (Bull. : Fr.) Murrill: Mirnoye, corticated L, diam. $37 \mathrm{~cm}$ (AGS 25853). 
Phellinus abietis (P. Karst.) Jahn: Mirnoye, living Psi, diam. $50 \mathrm{~cm}$ (HK 26384), living Psi, diam. $25 \mathrm{~cm}$ ( $H K$ 26385), dead, erect Psi, decay 2, diam. $24 \mathrm{~cm}$ ( $H K 26386)$. The identity of these specimens was confirmed by DNA sequences.

Phellinus alni (Bondartsev) Parmasto: Mirnoye, $\mathrm{Al}(H K$ sight $)$.

Phellinus cinereus (Niemelä) M. Fischer: Mirnoye, dead erect B $\times 2(H K$ sight $)$.

Phellinus ferrugineofuscus (P. Karst.) Bourdot: Lebed': corticated Pic, diam. $20 \mathrm{~cm}$ (AGS 25942).

Phellinus laevigatus (P. Karst.) Bourdot \& Galzin: Mirnoye, decorticated B, decay 2, diam. 14 cm (HK 26338), dead, erect, corticated B, decay 2, diam. $5 \mathrm{~cm}$ (HK 26380), corticated B, decay 2, diam. $14 \mathrm{~cm}$ (HK 26402).

Phellinus lundellii Niemelä: Bor, corticated Al, decay 2, diam. $5 \mathrm{~cm}$ (HK 26265), Al (HK sight).

Phellinus punctatus (P. Karst.) Pilát: Mirnoye, corticated Scap snag, decay 2, diam. $10 \mathrm{~cm}(H K$ 26412), Scap (HK sight).

Phellinus tremulae (Bondartsev) Bondartsev \& P.N. Borisov: Mirnoye, living Pop, diam. $15 \mathrm{~cm}$ (HK 26391), living Pop $\times 11$ (HK sight).

Piptoporus betulinus (Bull. : Fr.) P. Karst.: Mirnoye, corticated B, decay 3, diam. $4 \mathrm{~cm}(H K$ sight $)$, corticated $\mathrm{B} \times 15(H K$ sight $)$.

Polyporus ciliatus (P. Karst.) Sacc.: Mirnoye, Ssib branch, diam. $3 \mathrm{~cm}$ (AGS 25962).

Polyporus leptocephalus (Jacq. : Fr.) Fr.: Lebed, corticated Al, decay 2, diam. $3.5 \mathrm{~cm}$ (HK 26425).

Postia alni Niemelä \& Vampola: Mirnoye, decorticated Al, decay 2, diam. $7 \mathrm{~cm}$ (HK 26378).

Postia caesia (Schrad. : Fr.) P. Karst.: Lebed, decorticated Pic, decay 3, diam. $13 \mathrm{~cm}(H K$ 26440).

Postia leucomallella (Murrill) Jülich: Lebed': partly decorticated Pic, diam. $15 \mathrm{~cm} \mathrm{(AGS}$ 25895).
Postia placenta (Fr.) M.J. Larsen \& Lombard: Mirnoye, fallen, decorticated Pic, diam. $11 \mathrm{~cm}$ (AGS 25930).

Postia sericeomollis (Romell) Jülich: Bor, decorticated Psy, decay 3, diam. $23 \mathrm{~cm}$ (HK 26296).

Schizophyllum commune Fr.: Mirnoye (west shore): corticated S, diam. $3 \mathrm{~cm}$ (AGS 25884).

Skeletocutis amorpha (Fr.) Kotl. \& Pouzar: Mirnoye, partly corticated Psy, diam. $15 \mathrm{~cm}$ (AGS 25819).

Skeletocutis biguttulata (Romell) Niemelä: Bor, decorticated Psy, decay 2, diam. $21 \mathrm{~cm}$ (HK 26294).

Skeletocutis odora (Sacc.) Ginns: Lebed', corticated Pic, diam. $18 \mathrm{~cm}$ (AGS 25872).

Steccherinum ochraceum (Pers.) Gray: Mirnoye, corticated Al, decay 3, diam. $4 \mathrm{~cm}$ (HK 26323), corticated B branch, decay 4, diam. $0.6 \mathrm{~cm}(H K$ $26336 b)$, Lebed', corticated B, decay 3, diam. 4 cm (HK 26433).

Steccherinum tenuispinum Spirin, Zmitr. \& Malysheva: Mirnoye, corticated Al, decay 3, diam. $4 \mathrm{~cm}$ (HK 26374).

Trametes hirsuta (Wulfen : Fr.) Pilát: Mirnoye, dead, erect Pop, decay 2, diam. $10 \mathrm{~cm}$ (HK sight).

Trametes suaveolens (Fr.) Fr.: Mirnoye (west shore), corticated Scap, diam. $5 \mathrm{~cm}$ (AGS 25952).

Trametes versicolor (L. : Fr.) Pilát: Mirnoye, decorticated, Al, decay 2, diam. $7 \mathrm{~cm}$ (HK 26377), corticated, cut B branch, decay 2, diam. $3 \mathrm{~cm}$ (HK 26379), $\mathrm{Al} \times 2$ (HK sight).

Trechispora mollusca (Pers. : Fr.) Liberta: Mirnoye, dead Fomes fomentarius/B (HK 26401).

Trichaptum abietinum (Pers. : Fr.) Ryvarden: Bor, corticated Psy, decay 2, diam. $8 \mathrm{~cm}$ ( $H K$ 26272), Mirnoye, decorticated Pic, decay 3, diam. $18 \mathrm{~cm}$ (HK 26346), corticated Pic, decay 2, diam. $8 \mathrm{~cm}$ (HK 26382), Pic (HK sight). 
Trichaptum fuscoviolaceum (J.C. Schmidt : Fr.) Kreisel: Bor, corticated Psy, decay 1, diam. 13 cm (HK 26271).

Trichaptum laricinum (P. Karst.) Ryvarden: Mirnoye, corticated L, diam. $1.5 \mathrm{~cm} \mathrm{(AGS}$ 25800).

Trichaptum pargamenum (Fr.) G. Cunn.: Mirnoye, corticated B, decay 3, diam. $4 \mathrm{~cm}(H K$ 26404).

Tyromyces chioneus (Fr.) P. Karst.: Lebed', corticated B, diam. $3 \mathrm{~cm}$ (AGS 25945).

\section{Clavarioid fungi}

Artomyces pyxidatus (Pers.) Jülich (AGS 25877): Mirnoye, decorticated Psy, decay 2, diam. $20 \mathrm{~cm}$. It is exceptional to see this species on coniferous substrate.

Clavaria argillacea Pers.: Bor, on soil (AGS 25925).

Clavaria falcata Pers.: Mirnoye (west shore): on soil ( $A G S$ 25987), Mirnoye, on soil $\times 4(A G S$ sight).

Clavaria fragilis Holmsk.: Mirnoye, on soil, meadow (AGS 25828).

Clavaria fumosa Pers.: Lebed', on soil and mosses (AGS 25944).

Clavaria sphagnicola Pers.: Bor, on mosses (AGS 25830).

Clavariadelphus ligula (Schaeff.) Donk: Bor, Psy needles $(A G S$ 25848) $\times 4(A G S$ sight $)$, Mirnoye $\times 6(A G S$ sight $)$.

Clavariadelphus pistillaris (L.) Donk: Mirnoye, on soil between mosses $(A G S 25915) \times 11(A G S$ sight).

Clavariadelphus sachalinensis (S. Imai) Corner: Mirnoye, decaying Psy and Pic needles $\times 5$ (AGS sight), Lebed': (AGS 25861).
Clavariadelphus truncatus Donk: Lebed', soil under Pic (AGS 25940).

Clavicorona taxophila (Thom) Doty: Bor, decaying litter, wet habitat (AGS 25978).

Clavulina cinerea (Bull.) J. Schröt.: Bor, soil and mosses (AGS 25903).

Clavulina coralloides (L.) J. Schröt.: Bor, soil and mosses (AGS 25823), Mirnoye, soil (AGS sight).

Clavulinopsis corniculata (Schaeff.) Corner: Mirnoye, litter and mosses (AGS 25894).

Clavulinopsis helvola (Pers.) Corner: Bor, mosses (AGS 25970), Lebed': litter (AGS 25919).

Lentaria byssiseda Corner: Lebed', fallen Pic branch (AGS 25868).

Lentaria dendroidea (O.R. Fr.) J.H. Petersen: Mirnoye, mosses and dead Pic needles ( $A G S$ 25961), Lebed', L needles (AGS 25907).

Macrotyphula fistulosa (Holmsk.) R.H. Petersen: Mirnoye, fallen B twigs in mosses (AGS 25809).

Macrotyphula juncea (Alb. \& Schwein.) Berthier: Bor, fallen leaves and grass litter ( $A G S$ 25871), Lebed': fallen leaves ( $A G S$ 25958).

Multiclavula corynoides (Peck) R.H. Petersen: Mirnoye, soil covered by algae ( $A G S$ 25898).

Multiclavula vernalis (Schwein.) R.H. Petersen: Bor, soil covered with algae ( $A G S$ 25840).

Pterula gracilis (Desm. \& Berk.) Corner: Mirnoye (west shore), decaying leaves (AGS 25801), Lebed', decaying grasses ( $A G S 25885$ ).

Ramaria abietina (Pers.) Quél.: Mirnoye, Pic needle litter ( $A G S$ 25932).

Ramaria corrugata (P. Karst.) Schild: Lebed', Pic needle litter ( $A G S$ 25858).

Ramaria eosanguinea R.H. Petersen: Lebed', soil in mixed Pic, B forest (AGS 25917). 
Ramaria eumorpha (P. Karst.) Corner: Bor, mixed Pic, Psy needle litter ( $A G S$ 25834).

Ramaria flaccida (Fr.) Bourdot: Mirnoye, mixed Pic, Psy needle litter (AGS 25912).

Ramaria ochrochlora Furrer-Ziogas \& Schild (AGS 25982): Lebed', soil in Pic dominated forest.

Macroscopically similar to R. abietina, which is widely distributed in circumboreal zone, whereas $R$. ochrochlora grows almost solely in Europe (Ural Mountains is the most eastern point); however, once collected also in Russian Far East (Primorsk District, Terney, Plastun, coll. I. Parmasto 20.9.1990) and ones at the foothills of Altay Mountains (Kemerovo region, "Gornaya Shoria» Nature Park, coll. A. Shiryaev 27.8.2011). $R$. abietina mostly grows on needlelitter as a saprotroph, whereas $R$. ochrochlora has been collected only from the soil. It is a mycorrhiza-forming species, with long white rhizomorphs in soil and has a white basal tomentum on the solid stipe. The fruit bodies are also larger than those of $R$. abietina.

Ramaria stricta (Pers.) Quél. s.lato: Lebed', very decayed Psy trunk and needle litter $(A G S$ 25936).

Ramaria suecica (Fr.) Donk: Mirnoye, mixed Pic, Psy needle litter (AGS 25889).

Ramariopsis subarctica Pilát: Mirnoye, mosses (AGS 25951).

Ramariopsis tenuiramosa Corner: Mirnoye, soil and litter in meadow, under grasses and herbs (AGS 25815).

Typhula capitata (Pat.) Berthier: Bor, grasses (AGS 25980), Lebed', Calamagrostis (AGS 25878).

Typhula caricina P. Karst.: Mirnoye, Carex (AGS 25810).

Typhula crassipes Fuckel: Bor, fallen leaves and herbs (AGS 25929), Lebed', decaying herbs (AGS 25974).
Typhula culmigena (Mont. \& Fr.) Berthier: Mirnoye (west shore), decaying $\mathrm{S}$ leaves ( $A G S$ 25892), Mirnoye, dead herbs (AGS 25852).

Typhula erythropus (Pers.) Fr.: Mirnoye, decaying $\mathrm{B}$ and $\mathrm{S}$ leaves (AGS 25955).

Typhula hyalina (Quél.) Berthier: Bor, dead Equisetum stems (AGS 25920), Mirnoye, decaying grasses ( $A G S$ 25963).

Typhula lutescens Boud.: Mirnoye, decaying herbs and leaves (AGS 25844).

Typhula micans (Pers.) Berthier: Mirnoye, decaying herbs ( $A G S 25824)$.

Typhula phacorrhiza (Reichard) Fr.: Lebed', decaying leaves and herbs (AGS 25923).

Typhula sclerotioides (Pers.) Fr.: Mirnoye, dead stems of herbs (AGS 25950).

Typhula setipes (Grev.) Berthier: Mirnoye, decaying S leaves (AGS 25997), Lebed', decaying $\mathrm{B}$ leaves (AGS 25859).

Typhula spathulata (Corner) Berthier: Lebed', fallen $\mathrm{S}$ and Ssib twigs (AGS 25829).

Typhula struthiopteridis Corner: Mirnoye, dead petioles of Matteuccia struthiopteris (AGS 25967).

Typhula subvariabilis Berthier: Mirnoye, dead petioles of Ssib leaves (AGS 25901).

Typhula todei Fr.: Bor, dead petioles of Athyrium filix-femina (AGS 25836).

Typhula uncialis (Grev.) Berthier: Bor, dead Chamaenerion stems (AGS 25806), Mirnoye, Chamaenerion stems (AGS sight), Lebed', Chamaenerion stems (AGS 25926).

Typhula variabilis (Grev.) Berthier: Mirnoye, dead stems of herbs (AGS 25983). 


\section{Non corticioid, poroid or clavarioid fungi}

Cantharellus cibarius Fr.: Lebed', soil in mixed forest (AGS 25843).

Hericium coralloides (Scop. : Fr.) Pers.: Mirnoye, dead, erect, corticated Pop, decay 1, diam. $17 \mathrm{~cm}$ (HK 26408), Lebed'( $A G S$ 25807).

Hydnellum caeruleum (Hornem.) P. Karst.: Lebed', soil in Psy, Pic forest ( $A G S$ 25946).

Hydnellum concrescens (Pers.) Banker: Mirnoye, soil in Psy dominated forest ( $A G S$ 25867).

Hydnellum ferrugineum (Fr.) P. Karst.: Bor, soil in Psy dominated forest ( $A G S$ 25803).

Hydnellum peckii Banker: Lebed', soil in Pic dominated forest (AGS 25971).

Hydnellum suaveolens (Scop. : Fr.) P. Karst.: Bor, sandy soil on graveyard ( $A G S$ 25918).

Hydnum repandum L.: Bor, soil in mixed forest (AGS 25880).

Hydnum umbilicatum Peck: Lebed', soil in mixed forest ( $A G S$ 25833).

Sarcodon scabrosus (Fr.) P. Karst.: Lebed', soil in Pic dominated forest (AGS 25902).

Sarcodon squamosus (Schaeff.) Quél.: Bor, soil in Psy dominated forest ( $A G S$ 25973).

Thelephora terrestris Ehrh. : Fr.: Bor, decorticated Psy, decay 4, diam. $7 \mathrm{~cm}$ (HK 26298), sandy soil under pines (AGS 25900), Mirnoye, on young pine seedlings ( $A G S 25825)$.

\section{Results and discussion}

Altogether 211 species of aphyllophoroid fungi from 94 genera were found in this study, and all of them are new for the area, since there are no earlier studies. Together with the previous publication (Kotiranta \& Shiryaev 2015), the total number of aphyllophoroid species from the Central Siberian Biosphere Nature Reserve is 296. It is more than other protected Nature Reserves in the middle boreal subzone in Western or Eastern Siberia.

We compared our results with areas west (1, 2 ) and east $(3,4)$ of our research area, Bor as central point.

1. West Siberia, «Samarovsky Chugas» Nature Park, Khanty-Mansi Autonomous District, 293 species (Zalesov \& Stavishenko 2010, Shiryaev 2015). $61^{\circ} 02^{\prime} \mathrm{N}, 69^{\circ} 21^{\prime} \mathrm{E}, 1090 \mathrm{~km}$ W of Bor.

2. Sabun village, «Sibirskiye Uvaly» Nature Park, Khanty-Mansi Autonomous District, 234 species (Shiryaev 2002, 2015, Stavishenko 2003). $61^{\circ} 50^{\prime} \mathrm{N}, 83^{\circ} 32^{\prime} \mathrm{E}, 343 \mathrm{~km} \mathrm{~W}$ of Bor.

3. East Siberia, Stolbovoy, Krasnoyarsk Kray, Evenkia, 248 species (Kotiranta \& Shiryaev 2015). $61^{\circ} 35^{\prime} \mathrm{N}, 89^{\circ} 57^{\prime} \mathrm{E}, 100 \mathrm{~km}$ E of Bor.

4. Erbogachen village, Irkutsk province, 221 species (Shiryaev \& Muzika 2015). 61 ${ }^{\circ} 16^{\prime} \mathrm{N}$, $108^{\circ} 01^{\prime} \mathrm{E}, 950 \mathrm{~km} \mathrm{E}$ of Bor.

In this study, the largest genera are Typhula (17 species), Hyphodontia (9), Phellinus (8), Tubulicrinis (8), Ramaria (8), Botryobasidium (7), Clavaria (5), Hydnellum (5), Tomentella (5), Postia (5) and Phlebia (5). These 11 most species rich genera include 82 species, which is $38.9 \%$ of all the species. Similar results give the other localities in East- and West Siberia and the variation is very small (37.5-40.4\%) in contrary to European maritime climate where it is only around 33\%, and in ultra-continental Yakutia (Sakha Republic, Russian Far East) it is increasing till 46\% (Shiryaev 2014).

The largest genus Typhula includes $8.1 \%$ of all species, and the share is similar to West Siberia (Shiryaev 2015). In general, the percentage of the Typhula species is increasing with increasing continentality (Shiryaev 2014).

The three basic aphyllophoroid morphological forms (corticioid, poroid and clavarioid) include 199 species. It is $94.3 \%$ of the total number of species, whereas the less rich forms (cantharelloid, thelephoroid, stipitate hericioid, etc.) sum only 12 species altogether. The number of species of the three large forms is: corticioids 92 species $(42.6 \%)$, poroids 58 (28.5\%) and clavarioids $49(23.2 \%)$ of all species. The proportion of these life forms is relatively similar to comparison areas. However, the share of 
corticioid and clavarioid taxa is slightly higher than of polypores farther to the east. In temperate zone of Eurasia the situation seems to be the same: the more harsh conditions and high continentality, the more corticioids and clavarioids in comparison to poroids (Shiryaev et al. 2010, 2012, Shiryaev 2014). Corticioid/poroid ratio is 1.5 , and is practically the same between all five localities, but increases slightly with increasing continentality (Shiryaev et al. 2012, Shiryaev 2015). To sum this: the harsher the conditions are, the simpler are the fruit bodies of aphyllophoroid fungi.

The Red Data Book of Krasnoyarsk province (Stepanov 2012) contains 15 aphyllophoroid fungi. In our study we find six of them from the Central Siberian Nature Reserve, viz. Clavaria purpurea, Clavariadelphus ligula, C. pistillaris, C. truncatus, Hericium coralloides and Osteina obducta. Of these C. pistillaris and $O$. obducta have their closest occurrences about $700 \mathrm{~km}$ south of our study area and C. purpurea, C. truncatus and $H$. coralloides $300 \mathrm{~km}$ south in Yeniseisk town in southern boreal subzone. Clavariadelphus ligula was earlier collected also from middle boreal subzone, about 300 $\mathrm{km}$ east of our study area. Haploporus odorus, which is everywhere a rare polypore, was noted in Mirnoye five times in luxuriant, relatively old or virgin forest. Without large nature reserves, with diverse habitats and substrates, $H$. odorus will disappear.

Acknowledgements: The authors would like to express their deep gratitude to P.V. Kochkarev, director of Central Siberian State Biosphere Nature Reserve for his valuable help during the expedition. We are grateful to D.S. Pavlov, director of A.N. Severtsov Institute of Ecology \& Evolution (Moscow) and Boris Sheftel' for inviting us to stay and work in Mirnoye biological station. Also we would like to thank R. Petersen (Knoxville, TN) for his help and discussions of Ramaria ochrochlora. The authors thank warmly I.V. Stavishenko (Ekaterinburg) for help with the identification of some specimens. Special thanks go to N.P. Kutafjeva (Krasnoyarsk), J. Päivärinta and P. Rusanen (both from Helsinki) for the help in material collecting.

\section{References}

Eriksson, J., Hjortstam, K. \& Ryvarden, L. 1981: The Corticiaceae of North Europe 6: 1051-1276. - Fungiflora. Oslo.

Eriksson, J. \& Ryvarden, L. 1973: The Corticiaceae of North Europe 2: 60-286. - Fungiflora. Oslo.

Eriksson, J. \& Ryvarden, L. 1976: The Corticiaceae of North Europe 4: 549-886. - Fungiflora. Oslo.

Grosse-Brauckmann, H. 2003: Zwei neue CorticiaceenArten (Basidiomycetes, Aphyllophorales) an abgestorbenen Rubus-Arten. - Zeitschr. Mycol. 69: 93-99.

Harris, I., Jones, P.D., Osborn, T.J. \& Lister, D.H. 2014: Updated high-resolution grids of monthly climatic observations the CRU TS3.10 Dataset. - Int. J. Climatol. 34: 623-642. doi: 10.1002/joc.3711.

Kotiranta, H. \& Saarenoksa, R. 2005: The genus Athelopsis (Aphyllophorales, Corticiaceae) in Finland. - Ann. Bot. Fennici 42: 335-342.

Kotiranta, H. \& Shiryaev, A.G. 2015: Aphyllophoroid fungi (Basidiomycota) in Tunguska River basin, central East Siberia, Russia. - Karstenia (present issue).

Langer, E. 1994: Die Gattung Hyphodontia John Eriksson. - Bibliotheca Mycologica 154: 1-298.

Langer, G. 1994: Die Gattung Botryobasidium Donk (Corticiaceae, Basidiomycetes). - Bibliotheca Mycologica 158: 1-459.

Larsson, K.H. 1998: Two new species of Hyphoderma. Nordic Journal of Botany 18: 121-127.

Shherbina, S. 2009: Flora of vascular plants of Central Siberian State Biosphere Reserve and neighboring territories. - Turzaninowia 12(1-2): 71-241 (in Russian).

Shiryaev, A.G. 2002: Clavarioid fungi of the Nature Park «Sibirskiye Uvaly». Ecological studies of the eastern part of Sibirskiye Uvaly: Proc. National Park Sibirskie Uvaly. - Nizhnevartovsk 1: 69-79 (in Russian).

Shiryaev, A.G. 2014: Spatial differentiation of the clavarioid mycobiota in Russia: eco-geographical aspect. - Dissertation of Dr. Sci. Moscow State Univ., 2014. pp. 304.

Shiryaev, A.G. 2015: Addition to aphyllophoroid mycobiota of the West and Central Siberia: studies in local scale. - Proc. Orenburg State Pedagog. Univ. (in press, in Russian).

Shiryaev, A.G., Kotiranta, H., Mukhin, V.A., Stavishenko, I.V. \& Ushakova, N.V. 2010: Aphyllophoroid fungi of Sverdlovsk region, Russia: biodiversity, distribution, ecology and the IUCN threat categories. - Russian Academy of Science, Ural Division. Ekaterinburg. 304 pp.

Shiryaev, A.G., Mukhin, V.A., Kotiranta, H., Stavishenko, I.V., Arefjev, S.P., Safonov M.A. \& Kosolapov, D.A. 2012: The biodiversity of Aphyllophoroid fungi in Urals. - The Urals and neighbouring territories. Abstracts of international conference in Ekaterinburg: 311-313 (in Russian).

Shiryaev, A.G. \& Muzika, S.M. 2015: Aphyllophoroid fungi of Central Siberia: the local structure and complexes of the middle boreal subzone. - Proc. Irkutsk Agric. Acad. 68: 63-75 (in Russian). 
Stavishenko, I.V. 2003: Wood-decaying fungi from the south part of «Sibirskiye Uvaly» Nature Park: ecological studies of the eastern part of Sibirkiye Uvaly.Proc. National Park Sibirskie Uvaly, Nizhnevartovsk 2: 26-35 (in Russian).

Stepanov, N.V. (ed.) 2012: Red Data Book of Krasnoyarsk territory: the rare and endangered species of wild plants and fungi, $2^{\text {nd }}$ ed. - Krasnoyarsk, 2012. pp. 576.

Zalesov, S.V. \& Stavishenko, I.V. 2010: Flora and fauna of «Smarovsky Chugas» Nature Park. Xylotrophic wood-decaying fungi. - Ekaterinburg. Ural Forestry University. pp. 104 (in Russian). 